\title{
A New Demodulation Method for Mechanical Fault Feature Extraction based on LOD and IEE
}

\author{
Kang Zhang ${ }^{1}$, Xiaorui $\mathrm{Niu}^{2}$, Yunjiao $\mathrm{Ma}^{1}$, Xiangmin Chen ${ }^{1}$, Lida Liao ${ }^{1}$, Jiateng $\mathrm{Wu}^{3}$ \\ ${ }^{I}$ School of Energy and Power Engineering, Changsha University of Science and Technology, Changsha, 410114, China, \\ zhangkang513@163.com \\ ${ }^{2}$ The Big Data Centre, Mingyang Smart Energy Group Limited, Zhongshan, 528437, China \\ ${ }^{3}$ College of Mechanical and Vehicle Engineering, Hunan University, Changsha, 410082, China
}

The rolling bearing and gear fault features are generally shown as modulation characteristics of their vibration signals. The empirical envelope (EE) method is an accordingly common demodulation method. However, the EE method has the defects of over- and undershoot, which may lead to demodulation error. According to this, an envelope optimization algorithm -- empirical optimal envelope (EOE) is introduced into the EE method, and an improved empirical envelope (IEE) method is obtained to calculate the instantaneous amplitude and instantaneous frequency of mono-component modulation signal. Furthermore, aiming at the actual measured mechanical vibration signal has multi-component modulation feature, the IEE method is combined with an adaptive signal decomposition method -- local oscillatory characteristic decomposition (LOD) proposed by the author, thereby a new multi-component signal demodulation method based on LOD and IEE is proposed. The proposed method is compared with Hilbert transform (HT) and Teager energy operator (TEO) demodulation methods by the simulation signal and actual measured mechanical vibration signal. The results show that the demodulation effects including edge effects, negative frequency, over- and undershoot of the proposed method are significantly improved and can extract the rolling bearing and gear fault feature information clearly.

Keywords: Multi-component modulation signal, local oscillatory-characteristic decomposition, improved empirical envelope, mechanical vibration signal, demodulation analysis, fault feature extraction.

\section{INTRODUCTION}

When the fault of rotating machinery occurs, the fault vibration signal is usually a multi-component modulation signal, and the fault features are usually included in the modulation information. Therefore, to obtain the fault feature information, the original signal must be decomposed into mono-component signal first, and then demodulated [1], [2]. At present, the commonly used signal decomposition methods include wavelet transform (WT) [3], empirical mode decomposition (EMD) [4], local mean decomposition (LMD) [5], and so on. They have been widely used in fault signal decomposition [6]-[10]. But with the development of research, some defects have been found, such as lack of adaptability of WT [11], the problems of end effects [12], over- and undershoot [13], mode mixing [14] in EMD, and the problems of large amount of calculation, or local amplitude abrupt changes [15] in LMD. Recently, the authors proposed another new adaptive signal decomposition method -- local oscillatory characteristic decomposition (LOD) [16]. This method is based on the local time-scale characteristic of the signal itself, and by mathematical operations including differential, coordinate domain transform, and piecewise linear transform, enabling the self-adaptive decomposition of a multi-component signal into a series of mono-oscillation components (MOCs) whose instantaneous frequencies have physical meanings. Compared with the traditional signal decomposition method, the LOD method has the advantages of high computation efficiency and slight end effects. Since the LOD method was proposed, it has been used in gear, rolling bearing, and rotor system fault vibration signal decomposition, and achieved good results [17]-[19].

But similarly, the MOC component is necessary to be demodulated to get instantaneous amplitude and instantaneous frequency, to finally obtain the fault feature information. The typical signal demodulation methods include Hilbert transform (HT), Teager energy operator demodulation (TEO) [20], [21], etc. The HT method is the most commonly used mono-component signal demodulation method, but due to the inevitable windowing effects and the occurrence of non-integral periodic sampling, the demodulation results of HT will produce obvious edge effects and negative frequency problems [22]. The principle of the TEO method is simple, and there is no need to carry out complex number calculations, so the calculation amount is 
small. However, the TEO method is greatly affected by noise, and the smoothness of demodulation result is poor. Moreover, when the amplitude of signal is a transient function of time, the TEO method will produce a large error, or even cannot get the correct result [22]. By research, the local extreme point of the signal is the key to obtain accurate modulation information. Empirical envelope (EE) [23] method considers the information of each local extreme point of the analyzed signal, and has a good local description ability. In addition, it avoids the windowing effects of HT and the energy operator's sensitivity to noise in the calculation process. In theory, it can effectively extract the amplitude and frequency modulation information of the signal. However, the traditional EE method uses the cubic spline interpolation to obtain the empirical envelope of signal, which may lead to over- and undershoot problems and make the demodulation results distorted. Empirical optimal envelope (EOE) [24] is a new envelope estimation method. In the EOE algorithm, the envelope distance function is firstly defined, and then the accurately tangent points of signal and envelope are obtained through iterative optimization of the envelope distance. Thus, this not only ensures the signal and envelope tangency at accurate contact points, but also ensures that the upper and lower envelopes of signal do not cross, so as to obtain the empirical optimal envelope without over- and undershoot problems.

As mentioned above, the widely used traditional demodulation methods, such as HT, TEO, and EE demodulation may have negative frequency, edge effects, over- and undershoot problems, which affect the demodulation results. In addition, the actual signals in nature, such as measured mechanical vibration signals, are generally multi-component modulated signals, but the abovementioned traditional methods cannot demodulate the multicomponent modulated signals directly. Therefore, the main research questions of this paper are how to solve the problems of negative frequency, edge effects, over- and undershoot in the process of demodulation, and how to transform the mechanical vibration signal with multi-component characteristics into mono-component modulation signal, and then demodulate to obtain fault feature information.

According to the above analysis, in this paper, the EOE algorithm is introduced into the EE method to obtain an improved empirical envelope (IEE), which is used to demodulate the MOC components obtained by LOD decomposition, and obtain its instantaneous amplitude and instantaneous frequency. Thus, a multi-component signal demodulation method based on LOD and IEE is proposed. The proposed method is compared qualitatively and quantitatively with the HT and TEO methods through simulation signals. The results show that the overall demodulation effect of the proposed method is better than the other two methods. At the same time, according to the multicomponent modulation characteristics of rolling bearing and gear fault vibration signals, the proposed method is applied to the analysis of rolling bearing and gear fault vibration signals, and the results show that the proposed method can extract the fault features information of such signals effectively.
The rest of the paper will be organized as follows. Section 2 illustrates the defects of the EE method and the IEE method is introduced. In Section 3, the proposed approach based on LOD and IEE is described. The comparisons of simulation signal analysis among TEO, HT, and the proposed methods are discussed in Section 4. The analysis results from gear and rolling bearing fault vibration signals measured from a test bench are given in Section 5. Section 6 gives the conclusion.

\section{IMPROVED EMPIRICAL ENVELOPE METHOD}

\section{A. Defects of EE method}

EE method is a kind of typical mono-component signal demodulation method. The core idea of the EE method is to decompose the mono-component signal into a pure amplitude modulation (AM) signal and a pure frequency modulation (FM) signal. Then, the instantaneous amplitude (i.e., envelope) and the instantaneous frequency can be calculated from the pure AM signal and the pure FM signal, respectively. The specific steps of the EE method can refer to [23]. Therefore, the decomposition effect directly determines the accuracy of instantaneous amplitude and instantaneous frequency. But in the EE method, the cubic spline function is adopted, and the pure AM signal and pure FM signal of each mono-component signal are obtained by multiple iterations. So that, on the one hand, due to the continuity and smoothness of cubic spline interpolation curve, the obtained envelope may cross with the signal, thus the envelope error such as overshoot or undershoot will be produced, and on the other hand, the envelope error generated by each cubic spline interpolation will accumulate with the increase of iteration times, finally, which will lead to large demodulation error.

\section{B. Improved empirical envelope method based on EOE}

In order to solve the defects of the EE method mentioned above, it is necessary to avoid the intersection between the envelope and the signal. Accordingly, an optimized envelope method -- EOE algorithm is introduced. The essence of this method is to find through iterative way the accurately tangent points of the signal and the envelope based on the initial envelope that was obtained by traditional interpolation method, so as to ensure that the signal and the envelope are always tangent at the contact points, thus the occurrence of cross is avoided. The main steps of the EOE algorithm are as follows:

1. Determine all local maximum values $E_{\max }$ of signal $\mathrm{x}(\mathrm{t})$, and use the cubic spline function to interpolate $\mathrm{E}_{\max }$ to obtain the initial upper envelope function $\mathrm{e}_{\mathrm{uk}}(\mathrm{t})(k=0 \ldots \ldots$ $n$, where $n$ is the number of iterations).

2. The upper envelope distance function $d_{u k}(t)$ is defined as:

$$
\mathrm{d}_{\mathrm{uk}}(\mathrm{t})=\mathrm{x}(\mathrm{t})-\mathrm{e}_{\mathrm{uk}}(\mathrm{t})
$$

3. The local maximum values of $d_{u k}(t)$ are determined and expressed as $\mathrm{d}_{\mathrm{uk}}\left(\mathrm{t}_{\max }^{\mathrm{uk}}\right)$, and then the time series [ $\left.\mathrm{t}_{\max }^{\mathrm{uk}}, \mathrm{x}\left(\mathrm{t}_{\max }^{\mathrm{uk}}\right)\right]$ is interpolated by the cubic spline interpolation function, and the new upper envelope function $\mathrm{e}_{\mathrm{u}(\mathrm{k}+1)}(\mathrm{t})$ is obtained. 
4. Step 2 and Step 3 are repeated $n$ times until the number of the local maximum values $d_{u n}\left(t_{\max }^{\text {un }}\right) \neq 0$ of the upper envelope distance function is less than a predetermined threshold $\delta$, then the final empirical upper envelope function $\mathrm{e}_{\mathrm{un}}(\mathrm{t})$ is obtained.

5. Determine all local minimum values $\mathrm{E}_{\min }$ of signal $x(t)$, and use the cubic spline function to interpolate $E_{\min }$ to obtain the initial lower envelope function $e_{l k}(t), k=0$......n.

6. The lower envelope distance function $\mathrm{d}_{\mathrm{lk}}(\mathrm{t})$ is defined as:

$$
d_{l k}(t)=e_{l k}(t)-x(t)
$$

7. The local maximum values of $\mathrm{d}_{\mathrm{lk}}(\mathrm{t})$ are determined and expressed as $\mathrm{d}_{\mathrm{lk}}\left(\mathrm{t}_{\mathrm{max}}^{\mathrm{lk}}\right)$, and then the time series [ $\left.\mathrm{t}_{\max }^{\mathrm{lk}}, \mathrm{x}\left(\mathrm{t}_{\max }^{\mathrm{lk}}\right)\right]$ is interpolated by the cubic spline interpolation function, and the new lower envelope function $\mathrm{e}_{\mathrm{l}(\mathrm{k}+1)}(\mathrm{t})$ is obtained.

Step 6 and Step 7 are repeated $n$ times until the number of the local maximum values $d_{\ln }\left(t_{\max }^{\ln }\right) \neq 0$ of the lower envelope distance function is less than a predetermined threshold $\delta$, then the final empirical lower envelope function $\mathrm{e}_{\mathrm{lk}}(\mathrm{t})$ is obtained.

At present, overshoot or undershoot is a qualitative description based on whether the envelope intersects with the signal or whether the envelope is far away from the signal. But in the EOE method, it can be described quantitatively by the upper envelope distance and the lower envelope distance. Taking the upper envelope as an example, when $\mathrm{d}_{\mathrm{u}}\left(\mathrm{t}_{\text {max }}^{\mathrm{u}}\right)>$ 0 , it means that the signal and the empirical envelope cross each other, i.e., the undershoot will be produced; and when $\mathrm{d}_{\mathrm{u}}\left(\mathrm{t}_{\max }^{\mathrm{u}}\right)<0$, it means that the empirical envelope does not tightly surround the signal, that is, overshoot is produced. Ideally, the final upper and lower envelope functions $d_{u}(t)$ and $d_{l}(t)$ are less than or equal to 0 , and when $d_{u}\left(t_{\text {max }}^{u}\right)=0$ or $\mathrm{d}_{\mathrm{l}}\left(\mathrm{t}_{\text {max }}^{\mathrm{l}}\right)=0$, it means that $\mathrm{x}(\mathrm{t})$ is tangent to $\mathrm{e}_{\mathrm{u}}(\mathrm{t})$ or $\mathrm{e}_{\mathrm{l}}(\mathrm{t})$ at the time point $\mathrm{t}_{\max }^{\mathrm{u}}$ or $\mathrm{t}_{\max }^{\mathrm{l}}$, respectively. Thus it ensures the signal and envelope are always tangent at the contact points. In addition, the upper envelope $\mathrm{e}_{\mathrm{u}}(\mathrm{t})$ and the lower envelope $\mathrm{e}_{\mathrm{l}}(\mathrm{t})$ satisfy $\mathrm{e}_{\mathrm{l}}(\mathrm{t}) \leq \mathrm{x}(\mathrm{t}) \leq \mathrm{e}_{\mathrm{u}}(\mathrm{t})$, so as to ensure the envelope will not cut the signal, and the upper envelope and the lower envelope will not cross. Therefore, the EOE method can significantly reduce the over- and undershoot phenomena of the envelope.

An AM-FM signal $x_{1}(t)$ is constructed to illustrate the effects of the EOE algorithm.

$$
\begin{gathered}
\mathrm{x}_{1}(\mathrm{t})=\left[1+0.5 \cos \left(\frac{\pi \mathrm{t}}{100}\right)\right] \sin \left[\frac{\pi \mathrm{t}}{2}+2 \cos \left(\frac{\pi \mathrm{t}}{50}\right)\right] \\
+4 \sin \left(\frac{\pi \mathrm{t}}{1200}\right) \cos \left(\frac{3 \pi \mathrm{t}}{25}\right)+7
\end{gathered}
$$

Where $t \in[0,600] s$, the initial upper envelope function $\mathrm{e}_{\mathrm{u} 0}(\mathrm{t})$ and the upper envelope distance function $\mathrm{d}_{\mathrm{u} 0}(\mathrm{t})$ of signal $\mathrm{x}_{1}(\mathrm{t})$ are firstly calculated, as shown in Fig.1.a). It can be seen that the local maximum values of $\mathrm{d}_{\mathrm{u} 0}(\mathrm{t})$ are not aligned with the horizontal zero value line, especially the waveform in the period of 280 350 s and $450 \sim 580 \mathrm{~s}$. The waveform in $450 \sim 580 \mathrm{~s}$ is intercepted, as shown in Fig.1.b). It can be seen that when the local maximum values of $d_{u 0}(t)$ were greater than 0 , the envelope showed undershoot phenomena, and when the local maximum values of $d_{u 0}(t)$ were less than 0 , the envelope showed overshoot phenomena. These prove that the overshoot and undershoot phenomena can be described by the envelope distance function. Further, according to the steps of the EOE algorithm, the optimized $\mathrm{e}_{\mathrm{u}}(\mathrm{t})$ and $\mathrm{d}_{\mathrm{u} 2}(\mathrm{t})$ are obtained after two iterations, as shown in Fig.2.a). Similarly, the waveform between 450 580s is intercepted, as shown in Fig.2.(b). It can be seen that the local maximum values of $\mathrm{d}_{\mathrm{u} 2}(\mathrm{t})$ are equal to 0 , and no overshoot and undershoot phenomena appeared in the corresponding envelope, which shows the improvement effects of the EOE algorithm.
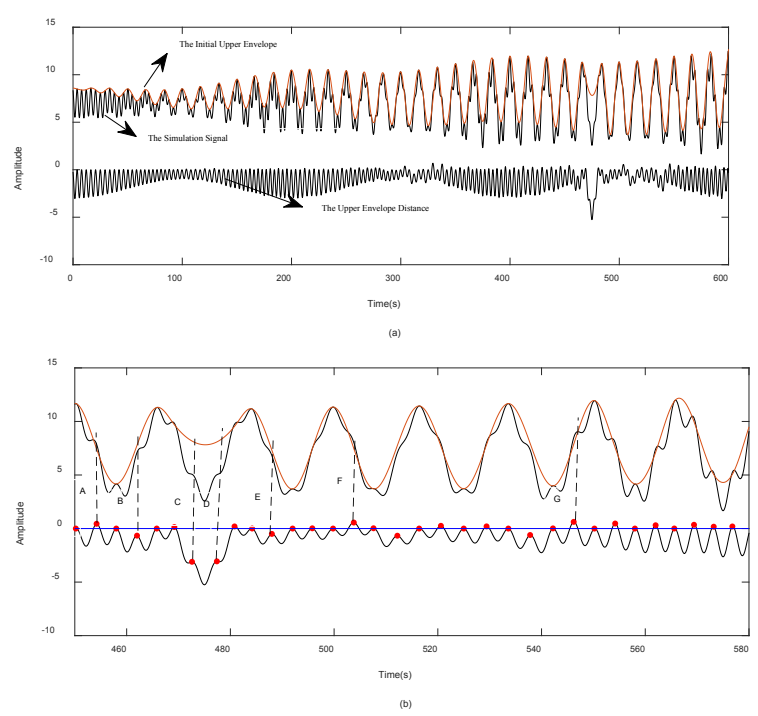

Fig.1. The initial upper envelope interpolation results a) The initial upper envelope and upper envelope distance b) The waveform between 450 580s.
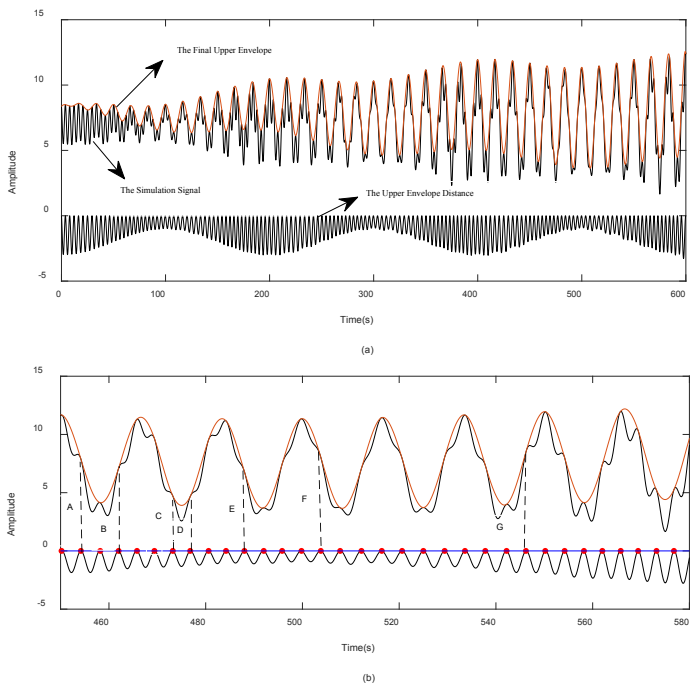

Fig.2. The final upper envelope interpolation results a) The final upper envelope and upper envelope distance b) The waveform between $450 \sim 580 \mathrm{~s}$. 
Using the EOE algorithm to optimize the envelope function obtained by the cubic spline interpolation in the EE method, the improved empirical envelope method (IEE) based on EOE can be obtained. Theoretically, more optimized envelope signal and pure FM signal can be calculated by the IEE method.

\section{A MULTI-COMPONENT MODULATION SIGNAL DEMODULATION METHOD}

For multi-component modulated signal such as the measured mechanical fault vibration signal, it is necessary to decompose it into the mono-component signal before demodulation. LOD is a new adaptive multi-component signal decomposition method. Based on the local extreme point of signal, this method can decompose a multicomponent signal into several mono-oscillation components (MOCs) by mathematical operations including differential, coordinate transform, and piecewise linear transform. The specific decomposition process of LOD and its advantages can refer to [11]. Then the IEE method is applied to calculate the instantaneous amplitude and instantaneous frequency of each MOC component, and the complete demodulation of the multi-component modulated signal can be realized. Based on this, a multi-component modulation signal demodulation method based on LOD and IEE is proposed. Let $y(t)$ be a multi-component modulation signal, and the specific steps are as follows:

1. $y(t)$ is decomposed into several MOC components and a residue by the LOD method:

$$
y(t)=\sum_{p=1}^{n} \operatorname{MOC}_{p}(t)+r_{n}(t)
$$

2. The EOE algorithm is used to optimize all empirical envelope functions of each MOC component.

3. According to the IEE method, the optimized empirical envelope function is used to normalize the MOC components, and then the instantaneous frequency and envelope signal of each MOC component are obtained.

\section{THE SIMULATION SIGNAL ANALYSIS}

Consider a multi-component AM-FM signal $\mathrm{x}_{2}(\mathrm{t})$ :

$$
\begin{gathered}
x_{2}(t)=3[1+0.2 \cos (2.5 \pi t)] \sin \left(50 \pi t+15 \pi t^{3}\right) \\
+6 t \sin (20 \pi t)
\end{gathered}
$$

$\mathrm{x}_{2}(\mathrm{t})$ is composed of an AM-FM component and an AM component. The sampling frequency is $1000 \mathrm{~Hz}$ and the sampling points are 2048. The time-domain waveform of $\mathrm{x}_{2}(\mathrm{t})$ and its LOD decomposition results are shown in Fig.3. It can be seen that the two MOC components correspond well to the two components of the original signal, indicating the adaptive decomposition characteristics of LOD. The theoretical instantaneous amplitudes and instantaneous frequencies of the two components of $x_{2}(t)$ are shown in Fig.4. The instantaneous amplitudes and instantaneous frequencies of the two MOC components calculated by the IEE method are shown in Fig.5. The threshold $\delta=1$ is set during the iteration. It can be seen that the calculated instantaneous amplitudes and instantaneous frequencies are consistent with the theoretical instantaneous amplitudes and instantaneous frequencies, and the curves are smooth and the edge effects are insignificant. For comparison, the HT method and the TEO method are used to calculate the instantaneous amplitudes and instantaneous frequencies of $\mathrm{MOC}_{1}$ and $\mathrm{MOC}_{2}$, and the results are shown in Fig.6. and Fig.7., respectively. Comparing with Fig.5., it can be seen that the modulation information of the two MOC components can also be obtained by the HT method and the TEO method. But the instantaneous amplitudes and instantaneous frequencies of $\mathrm{MOC}_{1}$ and $\mathrm{MOC}_{2}$ obtained by the HT method both produce obvious edge effects, and the instantaneous frequencies have unexplained negative frequency. The edge effects of instantaneous amplitudes and instantaneous frequencies obtained by the TEO method are reduced, but the smoothness of the instantaneous amplitudes and instantaneous frequencies is poor. Therefore, in conclusion, the instantaneous amplitudes and instantaneous frequencies obtained by the IEE method are the closest to the theoretical situation, and the edge effects and smoothness are also better than those obtained by the other two methods.
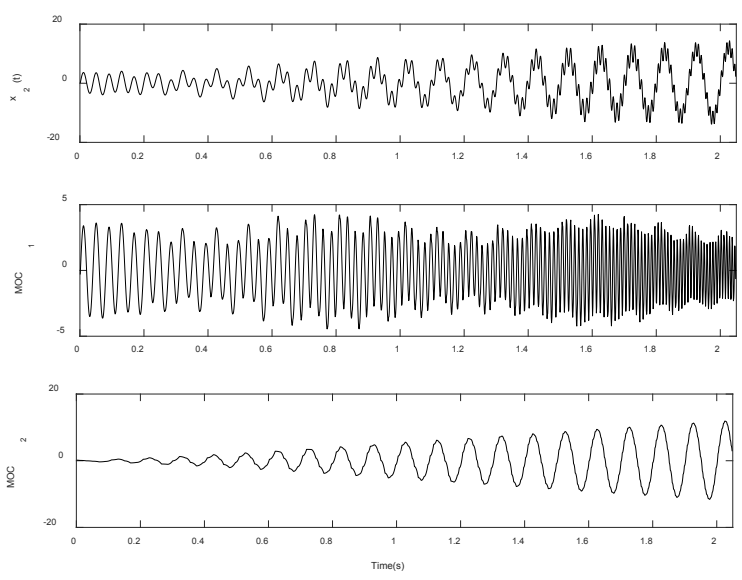

Fig.3. The waveform of simulation signal $x_{2}(t)$ and its LOD decomposition results.
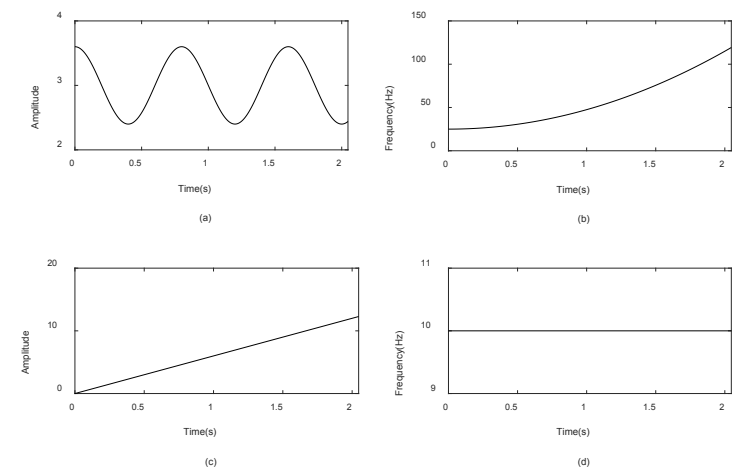

Fig.4. Instantaneous amplitude and instantaneous frequency of simulation signal $\mathrm{x}_{2}(\mathrm{t})$.

a), b) Instantaneous amplitude and instantaneous frequency of AMFM component

c), d) Instantaneous amplitude and instantaneous frequency of AM component. 


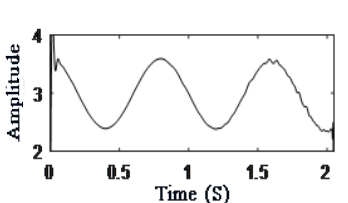

(a)

(c)

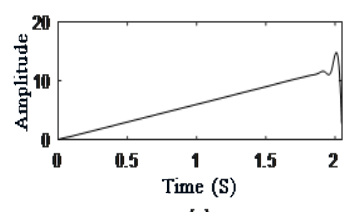

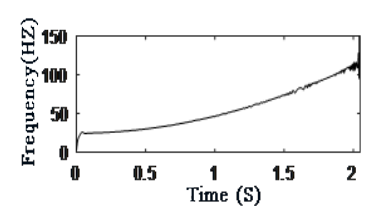

(b)

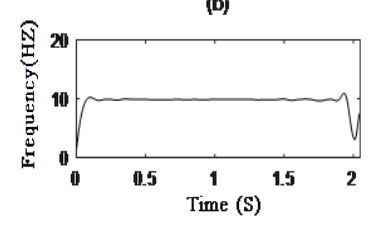

(d)
Fig.5. Instantaneous amplitude and instantaneous frequency of simulation signal $x_{2}(t)$ obtained by the IEE method

a), b) Instantaneous amplitude and instantaneous frequency of $\mathrm{MOC}_{1}$

c), d) Instantaneous amplitude and instantaneous frequency of $\mathrm{MOC}_{2}$.
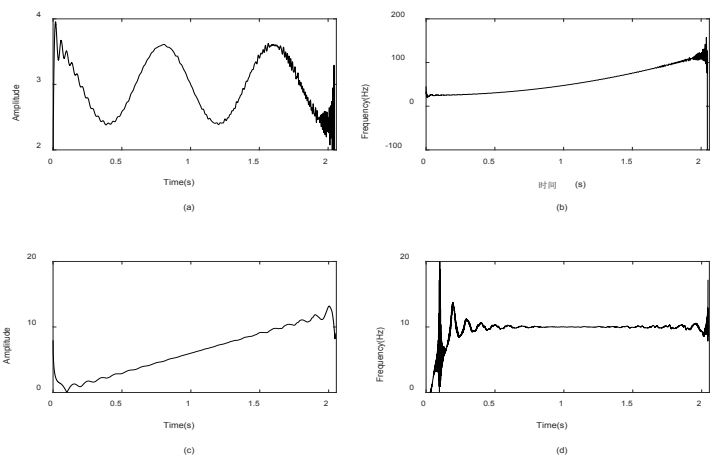

Fig.6. Instantaneous amplitude and instantaneous frequency of simulation signal $\mathrm{x}_{2}(\mathrm{t})$ obtained by the HT method

a), b) Instantaneous amplitude and instantaneous frequency of $\mathrm{MOC}_{1}$

c), d) Instantaneous amplitude and instantaneous frequency of $\mathrm{MOC}_{2}$.
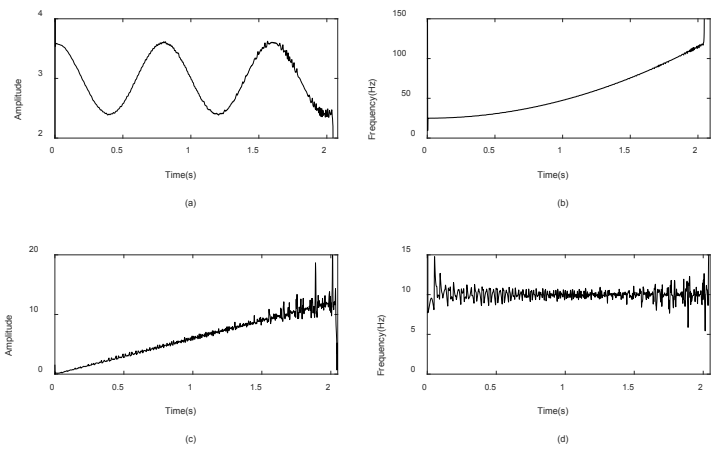

Fig.7. Instantaneous amplitude and instantaneous frequency of simulation signal $\mathrm{x}_{2}(\mathrm{t})$ obtained by the TEO method

a), b) Instantaneous amplitude and instantaneous frequency of $\mathrm{MOC}_{1}$

c), d) Instantaneous amplitude and instantaneous frequency of $\mathrm{MOC}_{2}$.
Furthermore, the instantaneous amplitudes and instantaneous frequencies of $\mathrm{MOC}_{1}$ and $\mathrm{MOC}_{2}$ obtained by the three methods are compared quantitatively by using the relative error index. The relative error index is defined as:

$$
\operatorname{err}=(\Delta / \mathrm{A}) \times 100 \%
$$

Where, A represents the theoretical instantaneous amplitude and instantaneous frequency of the signal, and $\Delta$ is the absolute error, that is, the absolute value of the difference between the calculated instantaneous amplitude and instantaneous frequency and the theoretical value. The relative error indices of instantaneous amplitude and instantaneous frequency of $\mathrm{MOC}_{1}$ and $\mathrm{MOC}_{2}$ obtained by the above three methods are shown in Table 1. It can be seen that the relative errors of instantaneous amplitudes and instantaneous frequencies of $\mathrm{MOC}_{1}$ and $\mathrm{MOC}_{2}$ components obtained by the IEE method are significantly smaller than those obtained by the HT method and the TEO method, which further prove the advantages of the IEE method.

Table 1. Relative errors of instantaneous amplitude and instantaneous frequency calculated by three methods.

\begin{tabular}{|l|l|l|l|}
\hline Relative error index & HT & TEO & IEE \\
\hline $\begin{array}{l}\text { Instantaneous } \\
\text { frequency of } \mathrm{MOC}_{1}\end{array}$ & 0.9990 & 0.9937 & 0.9902 \\
\hline $\begin{array}{l}\text { Instantaneous } \\
\text { amplitude of } \mathrm{MOC}_{1}\end{array}$ & 0.0105 & 0.0062 & 0.0067 \\
\hline $\begin{array}{l}\text { Instantaneous } \\
\text { frequency of } \mathrm{MOC}_{2}\end{array}$ & 0.9990 & 0.9936 & 0.2940 \\
\hline $\begin{array}{l}\text { Instantaneous } \\
\text { amplitude of } \mathrm{MOC}_{2}\end{array}$ & 0.0196 & 0.0526 & 0.0186 \\
\hline
\end{tabular}

\section{APPLICATION IN MECHANICAL FAULT FEATURE EXTRACTION}

In the case of rolling bearing and gear fault, their vibration signals usually are multi-component AM-FM signals. In order to extract their fault features, it is necessary to demodulate them. As described above, the multi-component signal demodulation method based on LOD and IEE is particularly suitable for processing such signals. Therefore, we use this method to analyze the rolling bearing and gear fault vibration signals, and then the fault features will be extracted in this section.

The gear and rolling bearing fault experiments were carried out on the rotating machinery fault test bench as shown in Fig.8. The drive motor in the test bench is a DC servo motor. Both the driving gear and the driven gear are spur gear, and the number of teeth is 37 . One tooth on the driven gear is cut off by laser, which is used as the fault gear. In all four bearing housings, the SKF-6311 ball bearing is installed, which also use laser to cut crack on the inner or outer race of the bearing to simulate local fault. The key phases are measured by an eddy current sensor. The vibration signals are measured by a PCB three-axis piezoelectric acceleration sensor placed on the bearing housing. The data acquisition equipment is LMS 40-channels vibration and noise data acquisition system. 


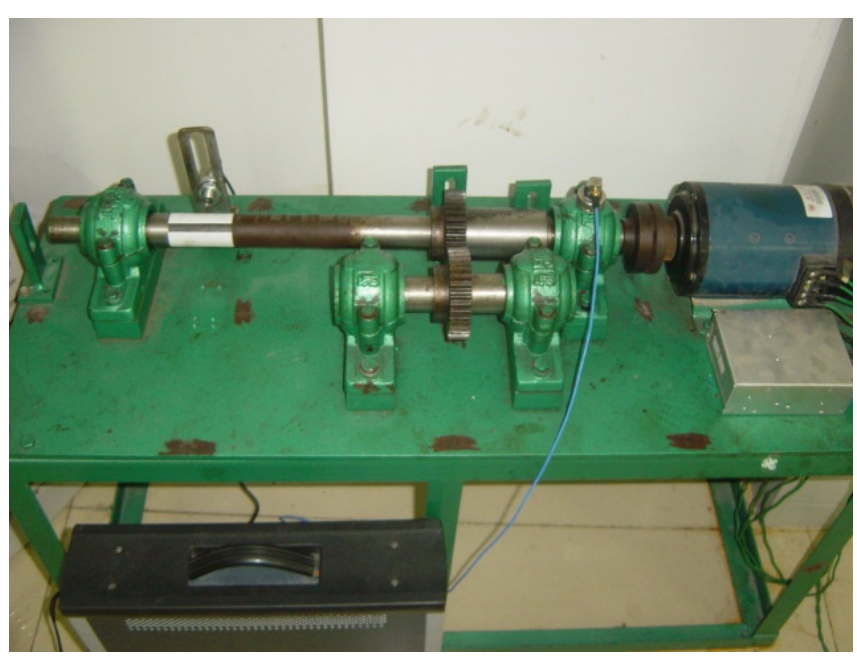

Fig.8. Rotating machinery fault test bench.

\section{A. Gear fault feature extraction}

In the gear fault experiment, the sampling frequency is $1024 \mathrm{~Hz}$ and the driven gear rotating frequency is $7 \mathrm{~Hz}$. The measured vibration signal of the driven gear fault is shown in Fig.9. According to the gear fault mechanism, the gear fault features are mainly embodied in the modulation sidebands with rotating frequency as interval on both sides of meshing frequency. The proposed method is used to demodulate this fault vibration signal and extract the fault features. Firstly, a total of seven MOC components were obtained by LOD decomposition. Since the highest analysis frequency is $512 \mathrm{~Hz}$ and the gear meshing frequency is $259 \mathrm{~Hz}$, theoretically, the signal only contains one time meshing frequency component, which is concentrated in the $\mathrm{MOC}_{1}$. The time domain waveform of $\mathrm{MOC}_{1}$ is shown in Fig.10. It can be seen that the $\mathrm{MOC}_{1}$ component has obvious modulation characteristics, and seven obvious periodic fluctuations occurred in one second, which just corresponds to the rotating frequency $7 \mathrm{~Hz}$. Instantaneous amplitude and instantaneous frequency of $\mathrm{MOC}_{1}$ are further calculated by the IEE method, and the results are shown in Fig.11. It can be seen that the obtained instantaneous amplitude and instantaneous frequency do not possess edge effects and negative frequency, and have good smoothness. The instantaneous frequency fluctuates around the meshing frequency $259 \mathrm{~Hz}$, while the instantaneous amplitude accurately reflects the amplitude modulation features of $\mathrm{MOC}_{1}$. The spectrum of instantaneous amplitude is shown in Fig.12. It can be seen that there is an obvious spectral line near the rotating frequency $7 \mathrm{~Hz}$, indicating that the gear has a local fault, which is consistent with the actual situation and verifies the effectiveness of the proposed method.

For comparison, the HT method and the TEO method are also used to demodulate the $\mathrm{MOC}_{1}$, respectively. The instantaneous amplitudes and instantaneous frequencies obtained by the two methods are shown in Fig.13. and Fig.14. As can be seen from Fig.13., the instantaneous amplitude obtained by the HT method can also reflect well the amplitude modulation characteristics of $\mathrm{MOC}_{1}$, but smoothness is slightly worse. The instantaneous frequency appears as a negative frequency phenomenon, which is obviously inconsistent with the reality. As can be seen from Fig.14., the smoothness of instantaneous amplitude and instantaneous frequency obtained by the TEO method is significantly worse than that obtained by the IEE method. In addition, the instantaneous frequency has significant distortion at the left end. Therefore, the comprehensive comparison shows that the IEE method has better demodulation effects than the HT method and the TEO method.

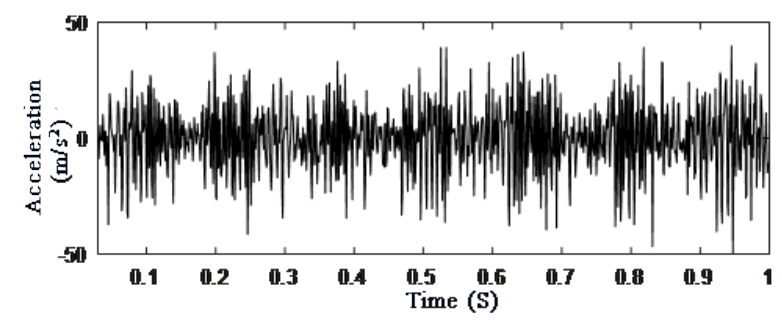

Fig.9. The gear fault vibration signal.

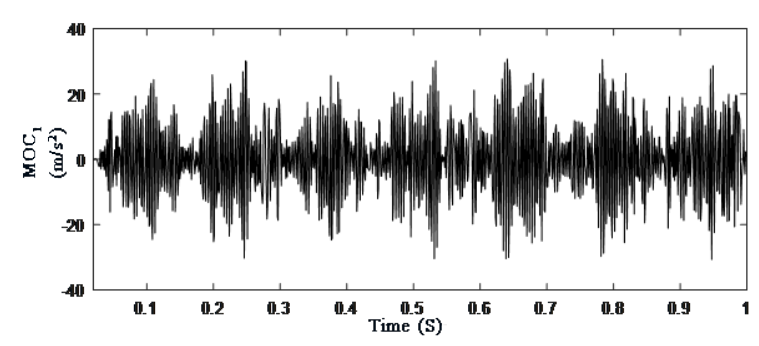

Fig.10. The $\mathrm{MOC}_{1}$ component of the gear fault vibration signal.

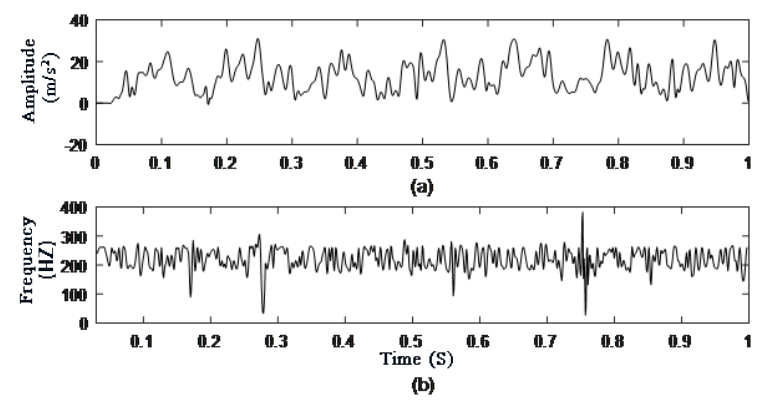

Fig.11. Instantaneous amplitude and instantaneous frequency of $\mathrm{MOC}_{1}$ obtained by IEE method.

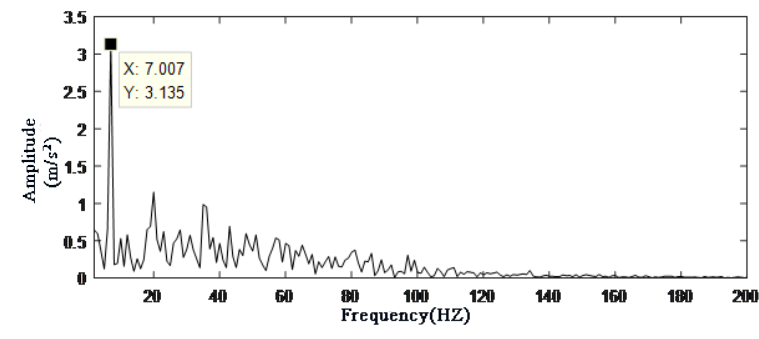

Fig.12. The spectrum of instantaneous amplitude. 


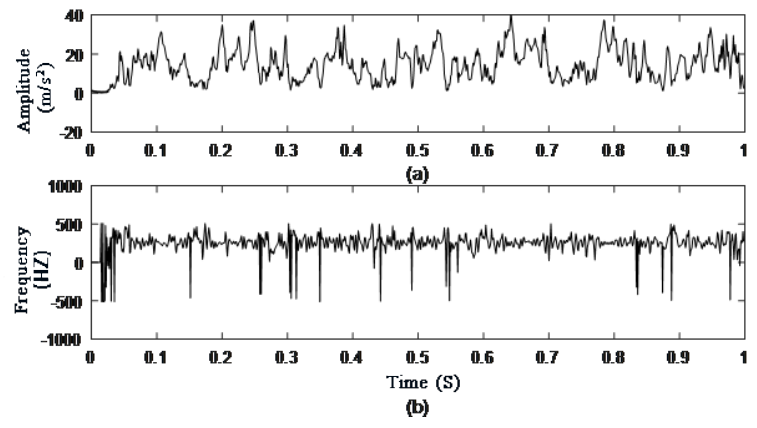

Fig.13. Instantaneous amplitude and instantaneous frequency of $\mathrm{MOC}_{1}$ obtained by HT method.
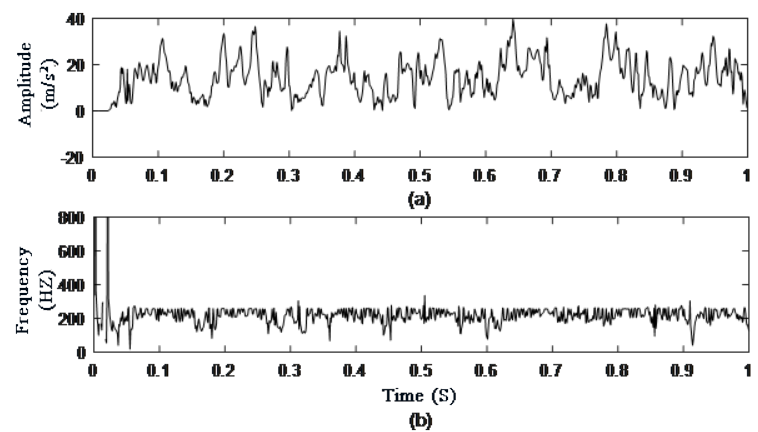

Fig.14. Instantaneous amplitude and instantaneous frequency of $\mathrm{MOC}_{1}$ obtained by TEO method.

\section{B. Rolling bearing fault feature extraction}

On the test bench shown in Fig.8., a crack fault is set on the inner race of a rolling bearing by laser cutting, and this rolling bearing is installed on the right end bearing housing of the driven gear shaft to carry out the rolling bearing fault experiment. The sampling frequency is $4096 \mathrm{~Hz}$, the rotating frequency is $20 \mathrm{~Hz}$, and the theoretical inner race fault feature frequency is $99.6 \mathrm{~Hz}$. The measured rolling bearing fault vibration signal and its spectrum are shown in Fig.15. and Fig.16., respectively. The rolling bearing inner race fault features are mainly presented as the modulation sidebands with the inner race fault feature frequency as interval on both sides of the bearing natural frequency. Meanwhile, the fault feature frequency is also modulated by the rotating frequency. Therefore, the method proposed in this paper can also be used for demodulation analysis to obtain the fault feature. Firstly, the original signal is decomposed by LOD. The LOD is an adaptive decomposition from high frequency to low frequency, and judging from the spectrum as shown in Fig.16. that the bearing natural vibration components are mainly distributed in the range of $[1100 \mathrm{~Hz} \sim 1200 \mathrm{~Hz}]$ ([400 Hz $\sim 600 \mathrm{~Hz}]$ are judged as gear meshing vibration components), the IEE is further used to demodulate the first MOC component $\mathrm{MOC}_{1}$ (as shown in Fig.17.). The instantaneous amplitude of $\mathrm{MOC}_{1}$ obtained by the IEE method is shown in Fig.18. It can be seen that the instantaneous amplitude smoothly and accurately reflects the amplitude modulation characteristic of $\mathrm{MOC}_{1}$. The spectrum of instantaneous amplitude is shown in Fig.19. It can be seen that there are obvious spectral lines near the inner race fault feature frequency $99.6 \mathrm{~Hz}$ and double rotating frequency $40 \mathrm{~Hz}$, which indicates the rolling bearing has inner race fault, and this is consistent with the actual situation.

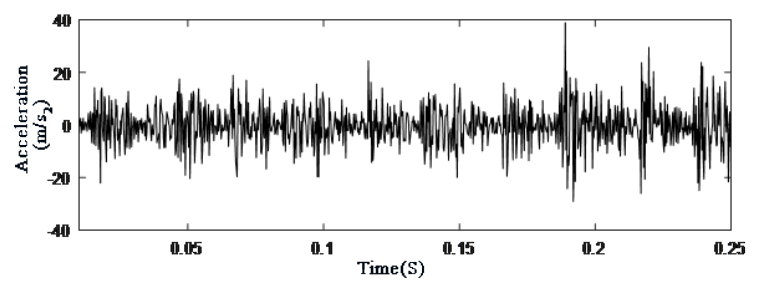

Fig.15. The vibration signal of rolling bearing with inner-race fault.

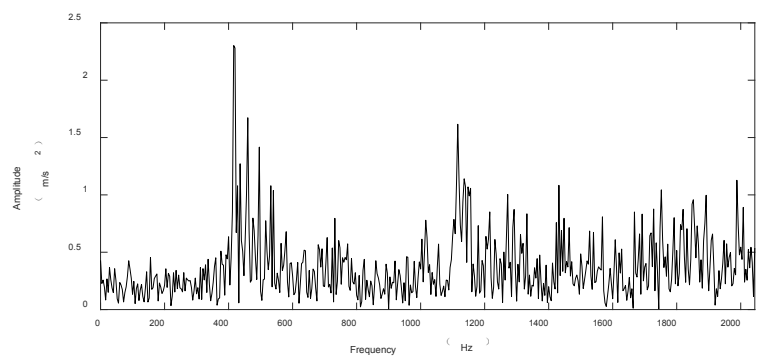

Fig.16. The spectrum of vibration signal of rolling bearing with inner-race fault.

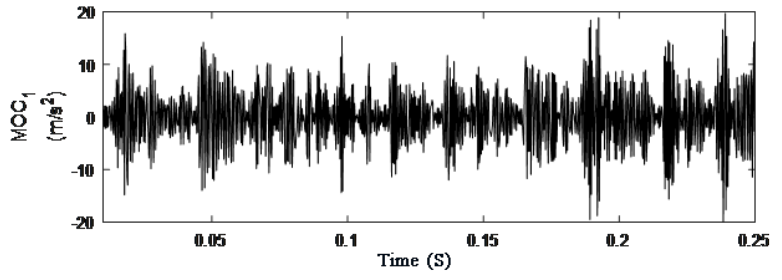

Fig.17. The $\mathrm{MOC}_{1}$ component of the vibration signal of rolling bearing with inner-race fault.

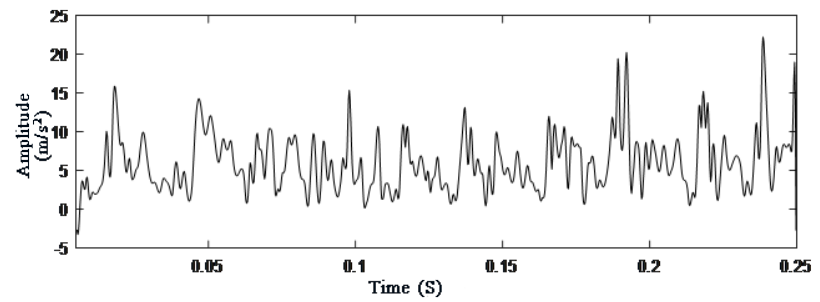

Fig.18. Instantaneous amplitude of $\mathrm{MOC}_{1}$ obtained by IEE method.

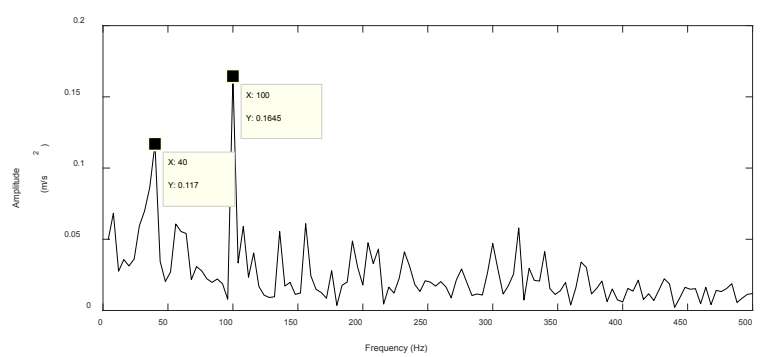

Fig.19. The spectrum of instantaneous amplitude. 


\section{CONCLUSION}

In this paper, the EE method is improved by the EOE algorithm, then the improved empirical envelope method is used to demodulate the MOC components obtained by LOD decomposition, thus a multi-component signal demodulation method based on LOD and IEE is proposed. The proposed method is applied to the analysis of the simulation signal and mechanical fault vibration signals, and compared with the HT and TEO methods, the following conclusions are drawn:

1. In the EOE algorithm, the phenomenon of overshoot or undershoot can be effectively avoided by optimizing the defined envelope distance function, which lays a foundation for obtaining a more accurate envelope signal in the future.

2. The simulation signal analysis shows that the edge effects of instantaneous amplitude and instantaneous frequency obtained by the IEE method are insignificant, and the smoothness is good and there is no negative frequency. Qualitative and quantitative comparison show that the overall demodulation effects of the IEE method are better than the HT and TEO demodulation methods.

3. Through the analysis of rolling bearing and gear fault vibration signals, it is shown that the multi-component signal demodulation method based on LOD and IEE can effectively process such multi-component modulation signals, and the corresponding fault feature information can be extracted conveniently from the obtained modulation information.

\section{ACKNOWLEDGMENT}

This work is supported by the National Natural Science Foundation of China (No. 51908064), Hunan Provincial Natural Science Foundation of China (No. 2018JJ3541), and Project of Education Department of Hunan Province (No. 20B019), and the authors are grateful to all reviewers and editors for their valuable comments.

\section{REFERENCES}

[1] Cheng, J., Yu, D., Yang, Y. (2004). Energy operator demodulating approach based on EMD and its application in mechanical fault diagnosis. Journal of Mechanical Engineering, 40 (8), 115-118.

[2] Zhang, K., Cheng, J. (2015). Roller bearing envelope analysis method based on local mean decomposition and kurtogram. Journal of Aerospace Power, 30 (12), 3043-3050.

[3] Liu, M., Weng, P. (2019). Fault diagnosis of ball Bearing elements: A generic procedure based on timefrequency analysis. Measurement Science Review, 19 (4), 185-194.

[4] Huang, N.E., Shen, Z., Long, S.R., Wu, M.C., Shih, H.H., Zheng, Q., Yen, N.C., Tung, C.C., Liu, H.H. (1998). The empirical mode decomposition and the Hilbert spectrum for nonlinear and non-stationary time series analysis. Proceedings of the Royal Society A, 454 (1971), 903-995.

[5] Smith, J.S. (2005). The local mean decomposition and its application to EEG perception data. Journal of the Royal Society Interface, 2 (5), 443-454.
[6] Wang, Y., He, Z., Zi, Y. (2009). A demodulation method based on local mean decomposition and its application in rub-impact fault diagnosis. Measurement Science and Technology, 20 (2), 1-10.

[7] Liu, W.Y., Gao, Q.W., Ye, G., Ma, R., Lu, X.N., Han, J.G. (2015). A novel wind turbine bearing fault diagnosis method based on Integral Extension LMD. Measurement, 74, 70-77.

[8] Mohanty, S., Gupta, K.K., Raju, K.S. (2018). Hurst based vibro-acoustic feature extraction of bearing using EMD and VMD. Measurement, 117, 200-220.

[9] Wu, F., Qu, L. (2009). Diagnosis of subharmonic faults of large rotating machinery based on EMD. Mechanical Systems and Signal Processing, 23 (2), 467-475.

[10] Feng, Z., Zuo, M.J., Qu, J., Tian, T., Liu, Z. (2013). Joint amplitude and frequency demodulation analysis based on local mean decomposition for fault diagnosis of planetary gearboxes. Mechanical Systems and Signal Processing, 40 (1), 56-75.

[11] Zhu, X., Wang, Y., Zhang, Y., Yuan, J. (2018). Method of incipient fault diagnosis of bearing based on adaptive optimal Morlet wavelet. Journal of Vibration, Measurement \& Diagnosis, 38 (5), 1021-1029.

[12] Lei, Y., Lin, J., He, Z., Zuo, M.J. (2013). A review on empirical mode decomposition in fault diagnosis of rotating machinery. Mechanical Systems and Signal Processing, 35 (1), 108-126.

[13] He, Z., Shen, Y., Wang, Q. (2012). Boundary extension for Hilbert-Huang transform inspired by gray prediction model. Signal Processing, 92 (3), 685-697.

[14] Zheng, J., Cheng, J., Yang, Y. (2014). Partly ensemble empirical mode decomposition: An improved noiseassisted method for eliminating mode mixing. Signal Processing, 96, 362-374.

[15] Ren, D. (2008). Study on methods and system for fault characteristics extraction of rotating machines based on local mean decomposition. Published doctoral dissertation, Zhejiang University, Hangzhou, China.

[16] Zhang, K., Chen, X., Liao, L., Tang, M., Wu, J. (2018). A new rotating machinery fault diagnosis method based on local oscillatory-characteristic decomposition. Digital Signal Processing, 78, 98-107.

[17] Zhang, K., Wu, J., Liao, L. (2015). Local oscillatorycharacteristic decomposition and its application in gear envelope analysis. Journal of Vibration Engineering, 28 (5), 846-854.

[18] Zhang, K., Shi, Y., Tang, M., Wu, J. (2016). Local oscillatory-characteristic decomposition and its application in roller bearing fault diagnosis. Journal of Vibration and Shock, 35 (1), 89-95.

[19] Wu, J., Yang, Y., Yang, X., Cheng, J. (2018). Fault feature analysis of cracked gear based on LOD and analytical-FE method. Mechanical Systems and Signal Processing, 98, 951-967.

[20] Han, T., Liu, Q., Zhang, L., Tan, A.C.C. (2019). Fault feature extraction of low speed roller bearing based on Teager energy operator and CEEMD. Measurement, $138,400-408$. 
[21] Xu, Y., Cai, Z., Ding, K. (2018). An enhanced bearing fault diagnosis method based on TVF-EMD and a highorder energy operator. Measurement Science and Technology, 29, 095108.

[22] Huang, N.E., Wu, Z.H., Long, S.R., Arnold, K.C., Chen, X.Y., Blank, K. (2009). On instantaneous frequency. Advances in Adaptive Data Analysis, 1 (2), 177-229.
[23] Zheng, J., Cheng, J., Yang, Y. (2012). A new instantaneous frequency estimation approach-empirical envelope method. Journal of Vibration and Shock, 31 (17), 86-90.

[24] Zhang, Q., Jia, L. (2018). [An empirical optimal envelope method based on interpolation]. China Patent No. CN108520207A. Beijing: China National Intellectual Property Administration.

Received March 28, 2021 Accepted May 28, 2021 